\title{
Apparent Extended Body Motions in Depth
}

\author{
Heiko Hecht and Dennis R. Proffitt \\ University of Virginia
}

\begin{abstract}
Five experiments were designed to investigate the influence of three-dimensional (3-D) orientation change on apparent motion. Projections of an orientation-specific 3-D object were sequentially flashed in different locations and at different orientations. Such an occurrence could be resolved by perceiving a rotational motion in depth around an axis external to the object. Consistent with this proposal, it was found that observers perceived curved paths in depth. Although the magnitude of perceived trajectory curvature often fell short of that required for rotational motions in depth (3-D circularity), judgments of the slant of the virtual plane on which apparent motions occurred were quite close to the predictions of a model that proposes circular paths in depth.
\end{abstract}

Long-range apparent-motion displays typically present a stimulus shape that is sequentially flashed in different locations. In such situations, the shape appears to move along a linear trajectory between the two positions. It is also possible to change the orientation of the shape as well as its locations; when this is done, a curved trajectory is observed in its apparent motion path (Foster, 1975). To date, such paths have been investigated in detail only for motions occurring in the picture plane. The present study was designed to investigate the generality of this curved trajectory finding for apparent motions in depth.

Without the application of some constraint, the number of possible apparent-motion trajectories between two stimuli is potentially infinite; however, usually only a small number of these possible paths are perceived (Figure 1). For typical displays consisting of two alternating points (Figure 1A), straight paths are almost always reported unless other paths are explicitly primed (Shepard \& Zare, 1983). This straight path could possibly indicate the application of a minimization constraint, one that minimizes displacement of the object's center (Wertheimer, 1912). Just as gravity constrains the falling of objects such that their centers of mass describe straight motions toward the earth, so too apparent-motion trajectories could be understood as a similarly constrained motion.

In fact, gravity turns out not to be the best analogy for the constraints inherent in perceiving apparent-motion trajectories. To understand why this is so, it is necessary to investigate

This research was supported by U.S. Air Force Grant AFOSR-870238, National Aeronautics and Space Administration Grants NCA2255 and NCA2-468, and a James McKeen Cattell Sabbatical Award to Dennis R. Proffitt.

We thank Stephen Jacquot for programming all interactive graphics displays; Michael Kubovy and David Gilden for valuable advice at various stages of this project; Frank Durgin, Ellen McAfee, and Jeannine Pinto for helpful criticism on earlier versions of this article; and David Foster, Walter Gerbino, Roger Shepard, and one anonymous reviewer for their thoughtful comments.

Correspondence concerning this article should be addressed to Dennis R. Proffitt, Department of Psychology, Gilmer Hall, University of Virginia, Charlottesville, Virginia 22903-2477. apparent motions between stimuli that realize not only translations but also orientation changes.

The role of orientability can be clarified by distinguishing between two classes of apparent motion contexts: particle and extended body motions (Proffitt, Gilden, Kaiser, \& Whelan, 1988). Particle-motion contexts are those in which the motions of the stimulus can be fully described in terms of the displacements of the shape's center. This can occur in two situations. First, whenever the shape has no intrinsic orientation, its motions reduce to those of its center. Similarly, the set of motions of an orientable shape that does not change in its orientation can be expressed in terms of its centroid's displacement. In contrast, extended body-motion contexts are all of those in which the shape changes its orientation. Unlike particle motions, extended body motions included rotations. Figure 2A shows an example of an extended body-motion context.

\section{Two Representations for Apparent Extended Body Motions}

Two alternative trajectories for apparent extended body motions can be given strong theoretical motivation. One alternative is depicted in Figure 2B. Here the centroid of the stimulus shape is seen moving linearly from one location to the other, and an apparent concomitant rotation about this center resolves the orientation change. As shown in Figure $2 \mathrm{C}$, the other alternative consists of a singular circular rotation of the shape. As discussed later, both of these alternatives could be described as minimal paths depending on the theory in which minimality is defined.

\section{Linear Centroid Trajectories With Concomitant Rotation}

Carlton and Shepard (1990a, 1990b) provided by far the most thorough discussion of different representations for apparent extended body motions. With respect to the linear motion alternative, they noted that this trajectory might be expected on the basis of a dynamical minimization principle; in particular, the law that natural motions minimize action (Energy $\times$ Time). In the absence of external forces, the least 


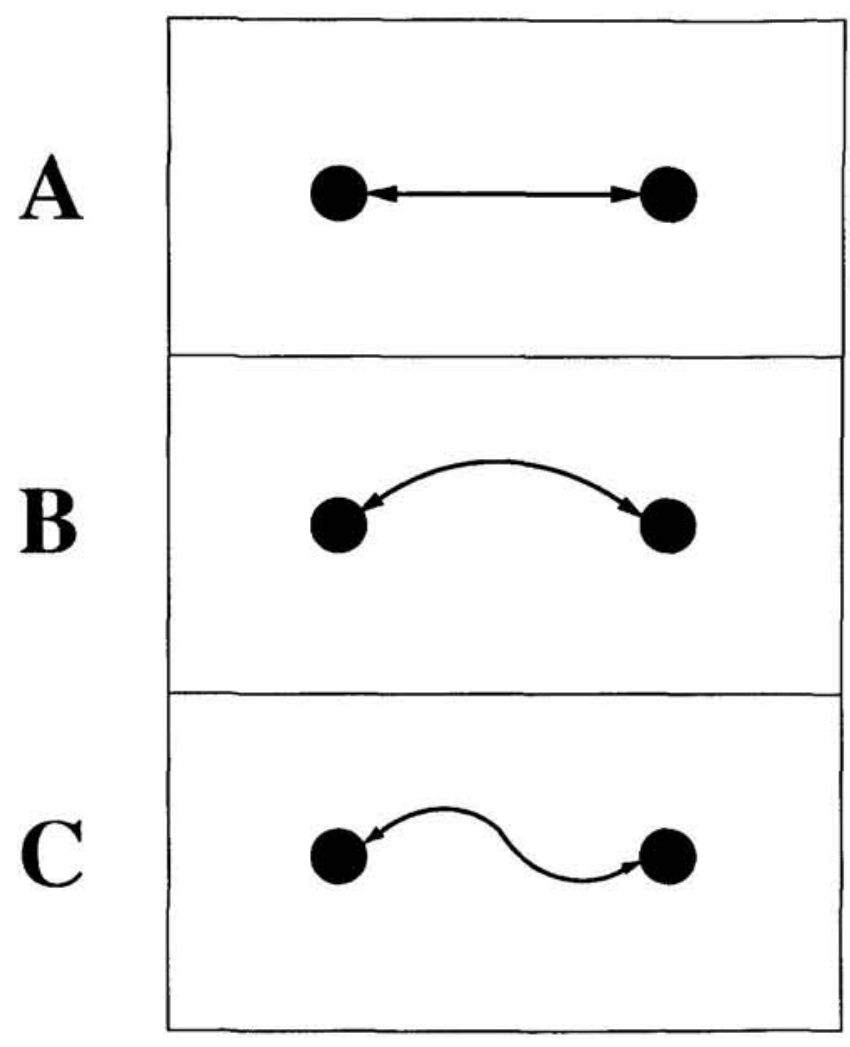

Figure 1. Different apparent-motion paths of point-like objects. (Typically observers report linear paths [Panel A]. Panels B and C depict other, more complex trajectories as examples of the indefinite number of apparent-motion paths that could be experienced.)

action path for a real object that is displaced and rotated is always achieved by a linear translation of its center of mass and a concomitant rotation about this point. Free-fall of a spinning object is an example of such a least action motion. The object will spin around its center of mass as this point falls straight down.

One kinematical representation of motion minimization also predicts a linear path for extended body motions. As discussed by Cutting and Proffitt (1982; see also Foster, 1978), the sum of all rotational motions is minimized to zero when rotations occur about an object's centroid. Moreover, the observer-relative common motion of an object is minimal when its corresponds to a linear path taken by some object point. Thus, by this representation of motion minimization, minimal extended body motions consist of centroid-relative rotations with concomitant centroid translations.

\section{Circular Trajectories}

Two other kinematical models of motion minimization prescribe circular paths for apparent extended body motions. The first model is based on the idea that a group of single rotations (SO[3]) can define the space of all possible three- dimensional orientation differences (Carlton \& Shepard, 1990a; Foster, 1975). Figure 2C shows one example of such a single rotation around a point external to the object. Chasles' theorem states that, for any object displacement and orientation change, there exists one axis in space about which the object can be rotated such that its initial position will be mapped into its final position. Such motions are minimal in the following sense: The surface of a sphere represents the manifold of possible three-dimensional orientations; thus, the shortest path (geodesic) between any two positions on this surface is an arc of a circle (Carlton \& Shepard, 1990a; Foster, 1978).

A second kinematical model of motion minimization, Restle's (1979) coding theory, also predicts circular apparent extended body motions. Restle proposed that the perceptual system seeks to minimize the number of information parameters needed to code an event. The number of variables that are needed to code an object's positions along its motion path is smaller for a circular path than it is for a straight path plus concomitant rotation. Given the initial position of the object, in the case of the circular path depicted in Figure 2C, only one angle of rotation has to be coded for every instant in time. For the path shown in Figure 2B, changes both in translation and rotation require coding for each new position.

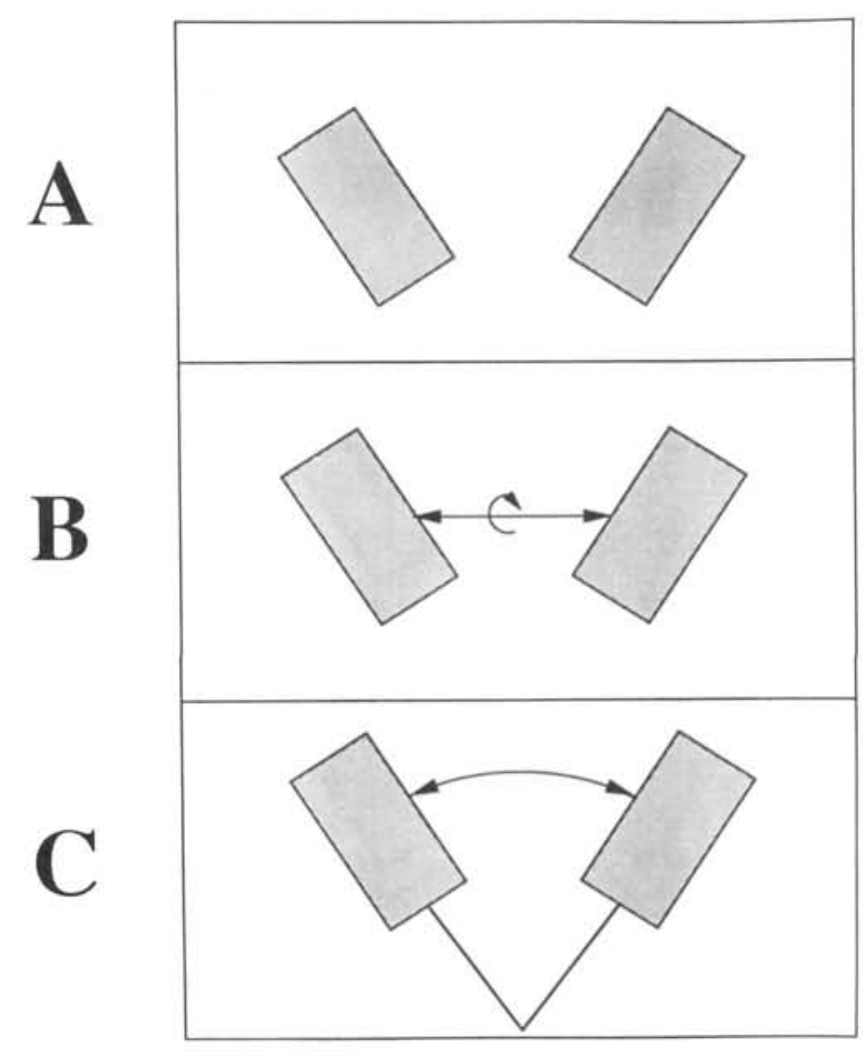

Figure 2. A stimulus pair of two differently oriented rectangles is represented in Panel A. (If they are flashed onto a screen in alternation, two motion paths could be predicted: a single circle [Panel C] or a linear common motion with concomitant rotation [Panel B].) 
Thus, the coding theory predicts circular apparent motion paths. ${ }^{1}$

\section{Empirical Evidence}

A number of studies have investigated the effect of orientation change on apparent motion trajectories (Bundesen, Larsen, \& Farrell, 1983; Farrell, 1983; Foster, 1975; McBeath, in press; McBeath \& Shepard, 1989; Mori, 1982; Proffitt et al., 1988). The finding common to these studies is that when stimuli are presented at different orientations, the motion perceived between them follows a curved path. The only qualification on this summary is that, when stimulus onset asynchronies (SOAs) are minimal, curved trajectories are not apparent (Farrell, 1983).

Foster (1975) first assessed apparent extended body-motion trajectories by having observers place a probe onto the apparent motion path. He found that the perceived trajectories corresponded to the single-rotation solution.

Proffitt et al. (1988) replicated this finding for a larger number of stimulus parameters. In all other cases, however, the perceived path was found to be curved but not circular. In particular, the amount of perceived curvature was found to depend on the following variables: (a) the amount of orientation change between the test stimuli, and (b) the orientation of stimulus symmetry.

The experimental situation used by Proffitt et al. (1988) had the spatial organization shown in Figure 3. The perceived motion path was assessed with a probe rectangle. It was flashed on the screen within the interstimulus interval (ISI), and it could be moved along an axis midway between the test stimuli. Subjects were instructed to place the probe on the perceived motion path.

Proffitt et al. (1988) found that the amplitude of perceived curvature was influenced by the magnitude of the orientation change between test stimuli. In Figure 3, this orientation change is defined by twice the angle $\theta$, and it was found that perceived trajectories approximated circularity only for angles $\boldsymbol{\theta}$ of $30^{\circ}$ or less. For larger angles, the extent of absolute perceived curvature increased; however, it fell increasingly short of circularity.

It was also found that the orientation of stimulus symmetry influenced the magnitude of perceived curvature. As is shown in Figure 3, $\alpha$ defines the orientation of the stimulus shape's major axis relative to the ray emanating from the origin of the single rotation solution through the test shape's centroid. Proffitt et al. (1988) found that when test stimuli were rotated ( $\alpha$ increased) such that the longitudinal axis of the test stimulus did not point toward the origin of the system, the amount of perceived curvature decreased. The larger the $\alpha$ (up to and including $90^{\circ}$ ), the shallower the perceived trajectory.

$\mathrm{McBeath}$ (in press) investigated the effects of orientation on the perceived trajectories of apparent-motion stimuli that consisted of symmetrical and asymmetrical two-dimensional polygons. Consistent with Proffitt et al.'s (1988) findings, larger orientation differences $(\Theta)$ led to increasingly shallower motion curves in relative terms. McBeath accounted for this finding with a quantitative model in which circular motions occurred on a plane that could be tilted in depth. The amount of tilt was a function of orientation magnitude $(\theta)$. Although the model provided a good fit for the data, the subjects in this study always reported that the polygons appeared to move in the picture plane.

To date, all published studies of apparent extended body motions have used stimulus arrangements that could be resolved with rotations in the picture plane. The present study sought to investigate whether these findings generalize to apparent extended body motions in depth. That is, will apparent-motion paths of stimuli that change orientation in planes other than the picture plane follow curved trajectories in three-dimensions as predicted by a circular path model?

\section{Apparent Motion in Depth}

Very little has been reported about the perception of depth in apparent-motion displays; moreover, there have been no direct assessments of apparent extended body-motion trajectories in depth. We now distinguish between two different situations that induce three-dimensional apparent motions.

\section{Phenomenal Depth From Cues (Textured Background or Disparity)}

In a number of experiments, Attneave and Block (1973) demonstrated that the perception of apparent motion was influenced by the background on which it occurs. Their displays always presented apparent motion between particles (two dots of light). In one condition, a regularly spaced grid indicated the frontoparallel surface. A small band around the dot's trajectory was left blank. In the experimental condition, this grid was drawn in perspective such that it suggested an oblique plane receding away from the observer into depth. The results showed that the optimal ISIs for the second condition were considerably longer compared with the first. That is, the stimulus needed more time to travel along the oblique plane even though physical and retinal stimulation was identical in both conditions.

Green and Odom (1986) demonstrated that perceived depth is an important factor in the process of correspondence matching. They used anaglyphic apparent-motion displays in which a stimulus was presented alternating with two stimuli that were displaced from it by the same two-dimensional distance but by different three-dimensional distances. When seen monocularly, correspondence was ambiguous. However, when viewing the same display stereoscopically, apparent motion occurred between those stimuli that were closest in threedimensional space.

\footnotetext{
' As was pointed out to us by Walter Gerbino, coding theory would give equal codings if only the changes in location and orientation between the two frames were being described. However, if continuous motion is coded, then the circular path alternative requires fewer descriptions than do the translation and concomitant rotation motions.
} 


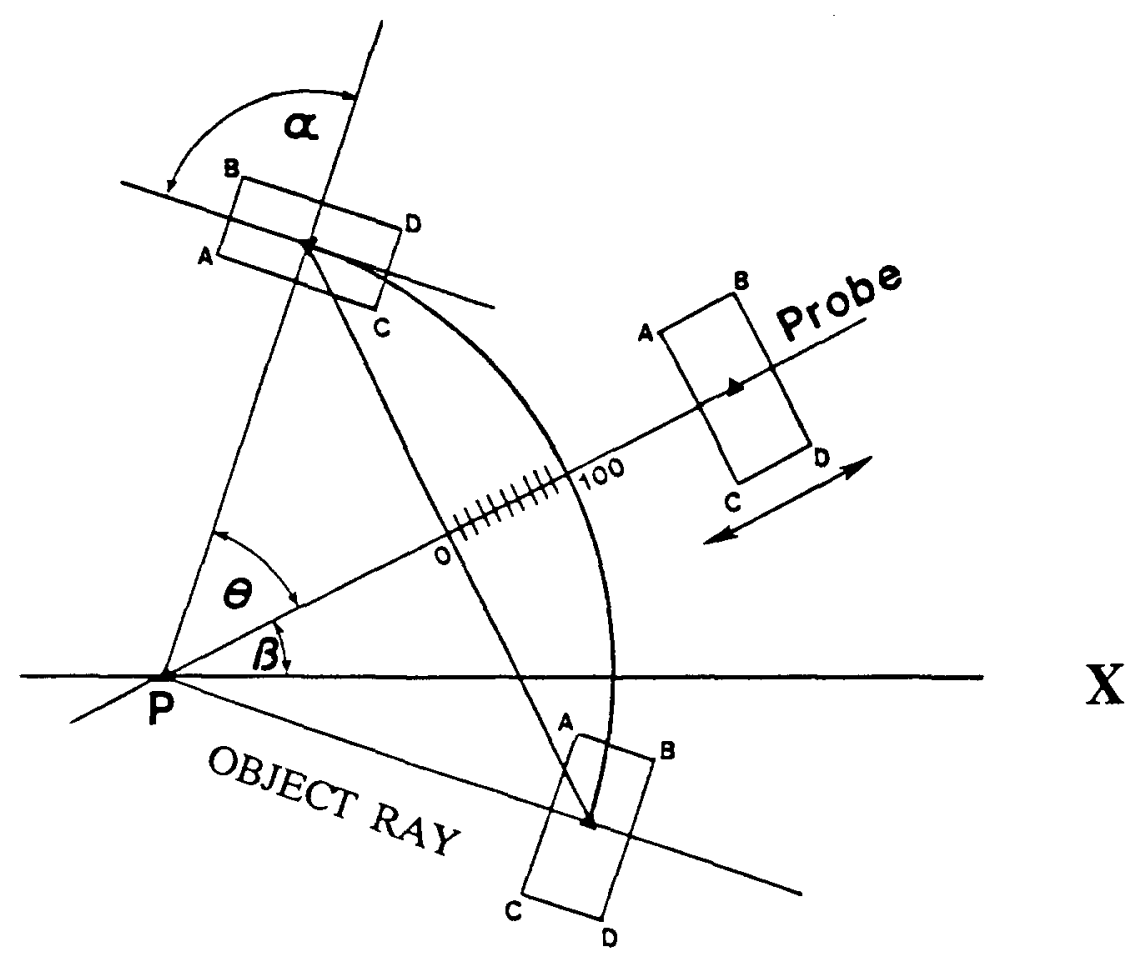

Figure 3. The stimulus parameters used by Proffitt et al. (1988, p. 467). (P is the origin of the coordinates; $\theta$, the angle between object ray and system axis; $\alpha$, the angle between the object's longitudinal axis and the object ray.)

\section{Phenomenal Depth From Three-Dimensional Orientation Change}

Apparent-motion displays that consist of two test stimuli with different contours are usually perceived as smooth plastic transformations from one shape to the other and back (Kolers \& Pomerantz, 1971). However, if the stimuli are identical but mirror reflected, a different phenomenon can be observed (e.g., Test Stimulus 1 is a pointed equilateral triangle, and Test Stimulus 2 is a mirror reflection of it around one of its sides). In this case, either a plastic transformation or a rotation in depth around the axis of reflection is perceived (Kolers \& Pomerantz, 1971; White, Wenderoth, \& Curthoys, 1979). Thus, orientation change can lead to apparent motion in depth. Shepard (1984) reported a similar finding: If the upper and lower halves of the contour of an ellipse were displayed in brief alternation, a "jump rope whirling around a horizontal axis" could be perceived (p. 428).

ISI has been shown to influence the quality of depth perception generated by apparent-motion displays. Gerbino (1984) found that, for a stimulus consisting of a triangle and its mirror reflection, with increasing ISIs the rotation into depth became more robust, peaking around $260 \mathrm{~ms}$. Kolers and Pomerantz (1971) concluded that "the visual system needs more time to construct a rigid change in depth than it needs to construct a co-planar change of shape" (p. 107). This finding held for stimuli that were reflections of trapezoidal objects, but not for stimuli that could not be mapped into one another. Those objects (e.g., square and circle) underwent plastic transformation in the picture plane. Shepard and Judd (1975) used apparent-motion displays of perspective drawings of wire-frame objects similar to the ones used by Shepard and Metzler (1971) in mental rotation tasks. They found that SOAs for which rigid apparent motion breaks down are proportional to the three-dimensional orientation difference of the objects (see also Farrell \& Shepard, 1981).

These studies show that motion in depth can be induced by orientation change. Apparent motion in depth may even be the preferred organization if the orientation change has a three-dimensional interpretation. Except for Shepard and Judd's (1975) study, however, the test stimuli were always confined to mirror reflections of one another, and the apparent trajectories in depth have never been reported. ${ }^{2}$

In sum, the following picture emerges: The perception of apparent extended body motion is governed by constraints that lead to the perception of curved trajectories, at least for orientation changes occurring in the picture plane. However, only for a limited range of display do the perceived trajectories approach circularity. Apparent extended body motions can occur in three-dimensions and, compared with traditional apparent-motion displays, they may seem more like the dy-

\footnotetext{
${ }^{2}$ A possible exception to this last claim is the work of McBeath (in press). However, this study used only two-dimensional stimuli that were rotated in the picture plane and that did not give rise to phenomenal apparent-motion paths in depth.
} 
namical object motions that occur in natural environments. Therefore, it is of interest to know whether the finding of curved paths generalizes to these motions in depth.

The present study was designed to investigate further the effects of three-dimensional orientation change on apparent extended body motions. Experiment 1 sought to examine whether simply making stimuli look three-dimensional would, by itself, change the apparent-motion paths. In this study, orientation changes could be resolved only in the picture plane. Experiment 2 was designed to examine the trajectories of apparent extended body motions in depth. Experiments 3 and 4 were designed to obtain an independent assessment of the orientation of the depth plane in which the curved apparent motions occurred. Finally, Experiment 5 sought to assess the influence of perspective projection on the magnitude of perceived curvature.

\section{Experiment 1}

This experiment was conducted to assess the apparentmotion trajectory of stimuli that could be interpreted as threedimensional objects. The experiment was designed to assess whether making stimuli look more like real three-dimensional objects would influence their apparent-motion path. Threedimensional objects might be more likely to evoke dynamically constrained apparent motions, that is, linear translations and concomitant rotations. Orientation changes were in the picture plane, but the stimuli themselves were oriented obliquely to it.

\section{Method}

Subjects. Twelve observers (6 men and 6 women) were tested individually. They were undergraduate students in introductory psychology classes at the University of Virginia and received partial course credit for their participation in the experiment.

Apparatus. A Tektronix 4129 3-D Color Graphics Workstation was used to display the stimuli. Observers sat in a dimly lit room facing the upright high-resolution $(4$ lines $/ \mathrm{mm})$ screen $(30 \times 35 \mathrm{~cm}$ wide).

Stimuli. Graphic images of domino-like objects were created. Top and end surfaces were marked with dots and lines, respectively, and the sides were color coded to provide clear information about the domino's orientation. Figure 4 shows the spatial organization of the apparent-motion display. The system was defined as the virtual triangular plane between the stimulus centers and the origin of the object rays (striped area).

The internal organization of the stimuli was described by the parameters $\alpha_{1}, \alpha_{2}$, and $\alpha_{3}$, which represent the amount of rotation around the internal domino-relative coordinate system, $x^{\prime}-, y^{\prime}-$, and $z^{\prime}$-axes. The parameters $\alpha_{1}$ or $\alpha_{2}$ changed in accordance with orthographic projections of real objects rotated around an internal axis. The $\alpha_{3}$ always remained zero; that is, the domino stimuli were aligned with their object rays.

The orientation of the whole system in virtual three-dimensional space was specified by the parameters $\beta_{1}, \beta_{2}$, and $\beta_{3}$, representing rotations of the system around the $x-, y$, and $z$-axes. Each stimulus pair consisted of two fixed-position test objects $(3.2 \times 1.6 \times 0.8 \mathrm{~cm})$ whose centers were $13.5 \mathrm{~cm}$ apart. The system was not rotated out of the frontoparallel plane, but the domino itself was.
The viewing distance of approximately $60 \mathrm{~cm}$ produced a visual angle of $12.7^{\circ}$ between the centers of the test dominos. This rather large spatial separation between the two test dominos was chosen for two reasons. First, this distance is comparable to that used by Proffitt et al. (1988). Second, it provided for sufficient space on the screen to differentiate maximally between possible outcomes. The origin of the system axis $(O)$ varied within a 4-cm circle at the center of the screen to lessen the likelihood that the screen center would serve as a cue for motion perception.

Two test dominos were flashed onto the screen alternatingly. The blinking sequence comprised $220 \mathrm{~ms}$ on-time for each domino, with an SOA of $500 \mathrm{~ms}$. Each cycle (Domino 1-ISI-Domino 2-ISI) was $1,000 \mathrm{~ms}$ long, causing an apparent back-and-forth motion. The stimulus durations and intervals were determined in pilot experiments to allow for sufficient time to perceive smooth apparent motion.

In addition to the two test dominos, a probe was introduced to allow subjects to indicate the amount of path curvature they perceived. It appeared within the ISIs and stayed on for $160 \mathrm{~ms}$. The probe could be moved continuously along the system axis. After the probe had been turned on by the observer, it appeared randomly at one or the other extreme end of the (invisible) system axis close to the border of the screen (this manipulation will be called direction). Two different probes were used: (a) a window, similar to that used by McBeath and Shepard (1989), consisting of two pointed triangles that were separated sufficiently to let the domino "pass through" it; and (b) a third domino at an intermediate position and orientation between the two dominos of the stimulus pair.

Design. The parameter $\beta_{3}$ (orientation of the system axis in the picture plane) and the direction of the probe were varied randomly. The angle between the system axis and either object ray, $\theta$, was $30^{\circ}$ throughout all conditions. $\beta_{3}$ varied randomly within each of the four $90^{\circ}$ sectors to minimize confounding effects that might be produced by the orientation of the display. $\beta_{1}$ and $\beta_{2}$ were 0 .

Angles $\alpha_{1}$ and $\alpha_{2}$ were varied systematically from $0^{\circ}$ to $60^{\circ}$ in $15^{\circ}$ steps leading to a block of 25 trials. Each trial was seen twice, once with a third domino serving as the probe and once with the window probe. Half of the subjects ( 3 men and 3 women) saw the block of 25 window probe trials first; the other subjects saw the domino block first. Trials were presented in randomized order within each block.

Procedure. Subjects were tested individually. The experimenter described apparent motion and the general experimental situation. Four practice trials were used to familiarize subjects with the task. They were told to turn the probe on only after a stable path was perceived (typically after 5-10 s in experimental trials). After having placed the probe, they hit a key to make a goodness rating scale appear at the bottom of the screen that could be adjusted continuously between the two ends marked "good" and "poor." For this rating, observers were instructed to give a rating of good when the probe placement seemed clear and easy. After one half of the trials, a short break was taken.

\section{Results}

In general, observers indicated motion paths that were very close to a circular motion around the origin $(\mathrm{O})$ of the system. The scores for probe placements were computed on a scale of 0 to 100 , with 0 representing a straight-line path between the centers of the test dominos and 100 representing a perfect circle around $\mathrm{O}$. For small $\alpha_{1}$ and $\alpha_{2}$ angles, the curvature of the path was slightly above 100; for large $\alpha_{1}$ and $\alpha_{2}$ angles, it was close to this score for a circle. The average placement of the probe, measured over all conditions, corresponded to $110.9 \%$ of the curvature for a circular path. Perceived curva- 


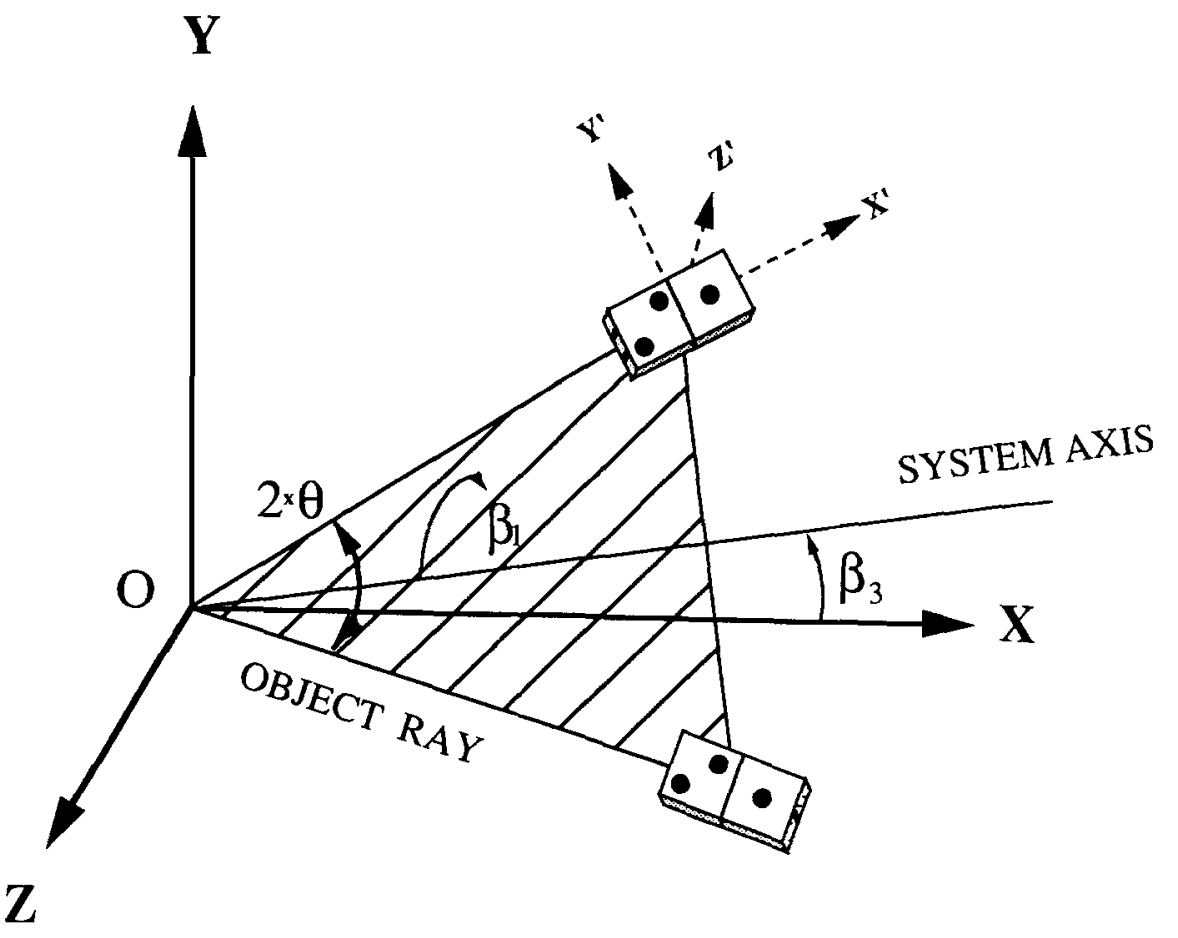

Figure 4. Cartesian coordinates for the apparent-motion stimuli used in all five experiments. (The external coordinate system $[x, y, z]$ is defined with respect to the screen. The internal coordinate system is defined with respect to the object itself. Its $x^{\prime}$ - and $y^{\prime}$-axes are in the plane formed by the two object centers and the origin [O]. The figure shows a rotation of the stimulus pair [system] around the external $x$-axis [angle $\beta_{1}>0$ ], but no rotation around the external $y$-axis $\left[\beta_{2}\right.$ is 0 and therefore does not appear in this figure]. $\theta$ is the angle between the object ray and the system axis.)

ture was significantly larger than circular, $t(11)=2.22, p<$ .05 .

Two repeated measures analyses of variance were performed. Probe placement or goodness rating were used as dependent variables, and $\alpha_{1}, \alpha_{2}$, gender, and probe style (domino vs. window) were used as independent variables. As shown in Figure 5, a slightly negative relation existed between $\alpha_{1}$ and $\alpha_{2}$ and both dependent measures. Angle $\alpha_{1}$ was significant for both dependent measures; for probe placement, $F(4$, $44)=5.09, p<.002$; for goodness rating, $F(4,44)=6.14, p$ $<.0005 ; \alpha_{2}$ was significant only for goodness rating, $F(4,44)$ $=5.48, p<.001$. Probe style, gender, and direction did not explain any significant amount of the variance in the dependent measures. The goodness ratings have been transformed to a scale from 0 (poor) to 100 (good).

\section{Discussion}

The results generally met the expectations of the circular path model. The motion paths between the two test dominos were curved and came fairly close to circularity. It seems that a possible three-dimensional interpretation of the stimulus and its resemblance to a real object does not lead to a deterioration of the percept of curved motion paths, given that $\boldsymbol{\theta}$ and SOA are chosen adequately. On the contrary, the curvature was more pronounced compared with the results of
Proffitt et al. (1988). In sum, the results suggest that apparentmotion stimuli, which can be interpreted as three-dimensional objects moving in the picture plane, are seen to follow curved trajectories similar to those perceived for two-dimensional objects.

\section{Experiment 2}

The purpose of this experiment was to investigate whether mere orientation changes within a stimulus pair could induce the perception of motion paths in depth. If so, would these trajectories follow the ellipses that result when circular paths in real space are projected onto the planar surface of the terminal's screen?

The previously mentioned path models can be applied to three-dimensional space: Any orientation change in a virtual three-dimensional space has a unique circular solution whose parallel projection corresponds to a segment of a particular ellipse on the screen. ${ }^{3}$ However, it had to be established whether observers perceived such apparent extended body motions as two- or three-dimensional. The circular path model produced two solutions for each display, one corre-

\footnotetext{
${ }^{3}$ Strictly speaking, this solution has to be helical, but for all situations examined in this study it is circular (see Carlton \& Shepard, 1990a, 1990b).
} 

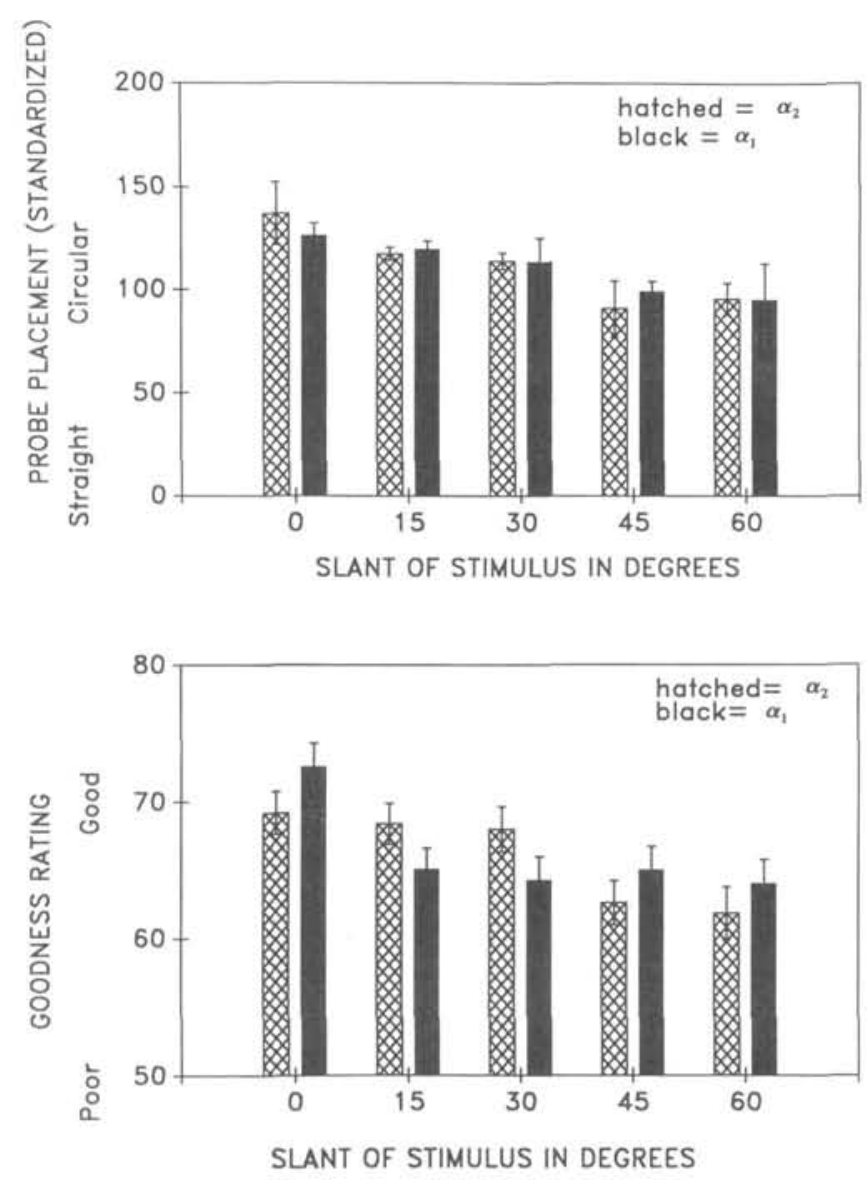

Figure 5. Results of Experiment 1. (Top panel: mean probe placements as a function of stimulus rotation around the internal $x^{\prime}$-axis $\left[\alpha_{1}\right]$ as well as around the internal $y^{\prime}$-axis $\left[\alpha_{2}\right]$. The scores were standardized such that a placement score of 0 corresponded to a straight-line path, and a score of 100 corresponded to a circular path on the projection screen. Bottom panel: mean goodness ratings as a function of the rotation of the stimulus around the internal $x^{\prime}$-axis $\left[\alpha_{1}\right]$ and the internal $y^{\prime}$-axis $\left[\alpha_{2}\right]$. A scale from $0[$ poor $]$ to 100 [good] was used.)

sponding to a three-dimensional interpretation and one corresponding to a two-dimensional interpretation.

Three types of stimuli were created: (a) stimulus pairs whose motion path allowed only a two-dimensional interpretation (motion in the frontoparallel plane); (b) stimulus pairs whose three-dimensional interpretations would project onto the screen as shallower curves; and (c) stimulus pairs whose threedimensional interpretations would project onto the screen as steeper curves. Figure 6 shows an example of each stimulus type with its linear and its curved (two-dimensional and threedimensional) interpretations. The three-dimensionally interpretable stimulus pairs were produced by rotating the stimulus system around the external $x$-axis (i.e., $\beta_{1}>0$ ) in the first case and around the external $y$-axis in the second case (i.e., $\beta_{2}>$ $0)$.

The two-dimensional solutions (circular path on the screen) were determined by the angle between the orientations of the longest linear edges of the dominos on the projection screen (two-dimensional $\Theta$ ) neglecting any depth interpretations. In other words, the domino was treated as a two-dimensional blob.

\section{2-D}

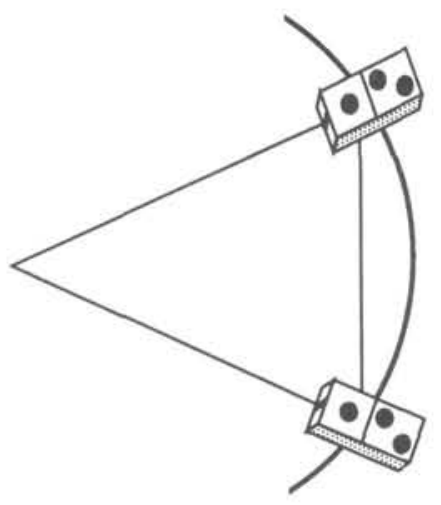

B1

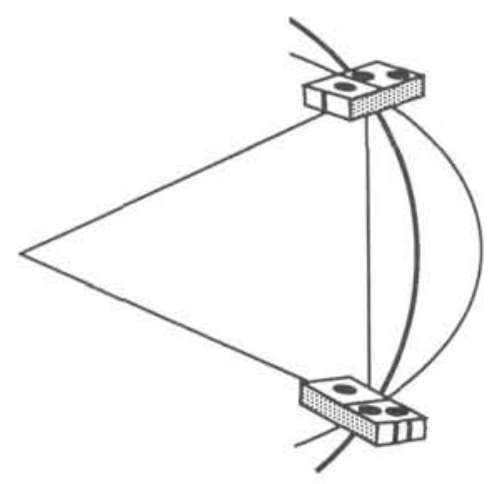

\section{B2}

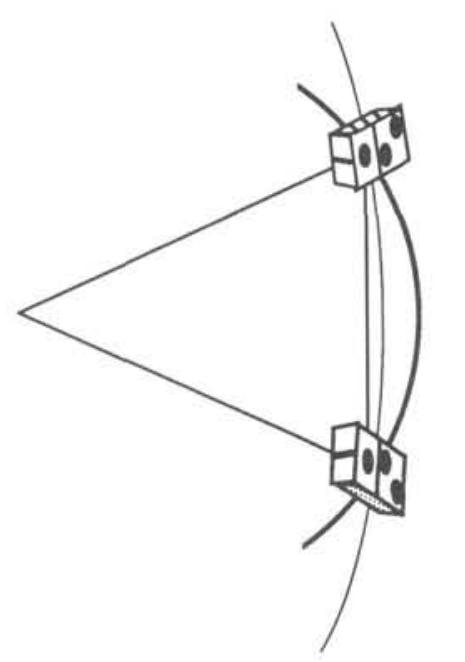

Figure 6. Ideal two-dimensional and three-dimensional solutions for experimental Conditions 2D, B1, and B2. (Thick lines indicate circular two-dimensional solutions; thin lines indicate straight and circular three-dimensional solutions. Note that in the top panel the domino is tilted internally [ $\alpha_{1}$ and $\alpha_{2}>0$ ] but its center stays in the picture plane.) 


\section{Method}

Subjects. Twelve observers (6 men and 6 women) were tested individually. They were undergraduate students in introductory psychology classes at the University of Virginia and received partial course credit for their participation in the experiment. All subjects had normal or corrected-to-normal vision.

Apparatus. The apparatus and viewing conditions were identical to those in Experiment 1.

Stimuli. The same domino-like objects were used as in Experiment 1 . However, because the system was rotated around the external $x$ - or $y$-axis, the necessary changes in projected shape were made (parallel projection). The fixed positions, blinking sequence of the test dominos, and timing remained unchanged. This would ensure direct comparability with the first experiment and allow sufficient SOAs for depth perception to occur. The value of $\theta$ was kept below $40^{\circ}$ such that the three-dimensional distance the domino had to travel did not enter the range for which Proffitt et al. (1988) found perceived curvature to decrease significantly from circularity. The probe, which could be moved along the system axis, participated in the apparent motion and reflected the object's orientation in the coordinate system. As in Experiment 1, both a window and a third domino were used for probes.

Design. Five stimulus pairs were created for each $\beta$ condition. Those stimulus pairs that had two-dimensional solutions only (Condition $2 \mathrm{D}$, i.e., all angles $\beta$ were 0 ) were $13.5 \mathrm{~cm}$ apart (center to center) and had orientation differences of $\theta=30^{\circ}$ or $35^{\circ}$. Stimulus pairs that produced steeper three-dimensional solutions (B1 Condition) varied by the rotation of the system around the external $x$-axis $\left(\beta_{1}\right.$ was $34.5^{\circ}, 36.1^{\circ}, 50.9^{\circ}, 62.3^{\circ}$, or $\left.72.2^{\circ}\right)$. Their respective angles (two-dimensional $\theta$ ) were $3.1^{\circ}, 7.1^{\circ}, 12.9^{\circ}, 20.6^{\circ}$, and $25.4^{\circ}$. Stimulus pairs with shallower three-dimensional solutions (B2 Condition) were rotated around the external $y$-axis by $48.8^{\circ}, 60.3^{\circ}, 61.0^{\circ}, 67.8^{\circ}$, or $72.2^{\circ}$; their angles (two-dimensional $\theta$ ) were $10^{\circ}, 15^{\circ}, 20^{\circ}, 25^{\circ}$, and $30^{\circ}$, respectively.

To make the stimuli in all three conditions look similarly solid, $\alpha_{1}$ and $\alpha_{2}$ were set to $20^{\circ}$ in Condition 2D. Otherwise, for $\alpha \mathrm{s}$ of 0 , only one main surface would have been visible. In all other conditions, $\alpha_{1}$ and $\alpha_{2}$ were 0 . The value of $\alpha_{3}$ was always 0 . The separation between the centers of the test dominos on the projection surface (D) was always $13.5 \mathrm{~cm}$. However, in virtual three-dimensional space this distance had to be decreased or increased (up to three times as large for the B1 Condition; see the Appendix for the method used to create the stimuli).

Procedure. The instructions for the subjects were identical to those given in Experiment 1. All 15 test stimuli were shown twice, once with the window (Block 1) and once with a third domino as the probe (Block 2). Six observers ( 3 men and 3 women) saw Block 1 first; the others saw Block 2 first. Within each block the trials were shown in random order. After it had been turned on by the observer, the probe could be moved along the system axis of the display. After each trial, the observers were asked to make two ratings.

The first rating concerned the difficulty of placing the probe onto the perceived motion path. In Experiment 1, subjects reported that, when rating goodness, they thought about it in terms of how difficult it was to place the probe. Thus, observers were asked to rate the difficulty to place the probe precisely onto the perceived motion path. The second rating assessed the amount of perceived depth of the motion path. It was explained that the path could either be seen parallel to the screen or receding into it. In the latter case, a depth rating should be made. The ratings were made by using a thumb wheel to move a marker along a continuous scale that was converted into a scale ranging from 0 to 100 . The difficulty scale was labeled easy and difficult; the depth scale had the labels in depth and in plane.

A short break was taken after the first half of the 30 trials. Latency times were recorded for the time it took to place the probe on the perceived motion path, but subjects were not told about this measure so they would not feel pressured to complete the task hastily.

\section{Results}

Figure 6 shows the solutions for the three conditions that correspond to the circular path model. For Condition 2D, there is only one circular path because it has only a twodimensional interpretation. For the other two conditions, however, the projection of a circular motion path in depth would result in a segment from the steep (B1) or the shallow (B2) part of an ellipse, whereas the two-dimensional solution for these stimuli would coincide with a path that is circular on the surface of the screen. The different solutions as well as the scores were computed in screen coordinates on a scale of 0 to 100 , with 0 corresponding to a straight-line path and 100 to a circular path (two-dimensional). That is, probe placements for all conditions were expressed in units on the projection surface relative to the scores for Condition $2 \mathrm{D}$.

The top panel of Figure 7 compares the ideal results for the parameters chosen for each condition with the actual probe-
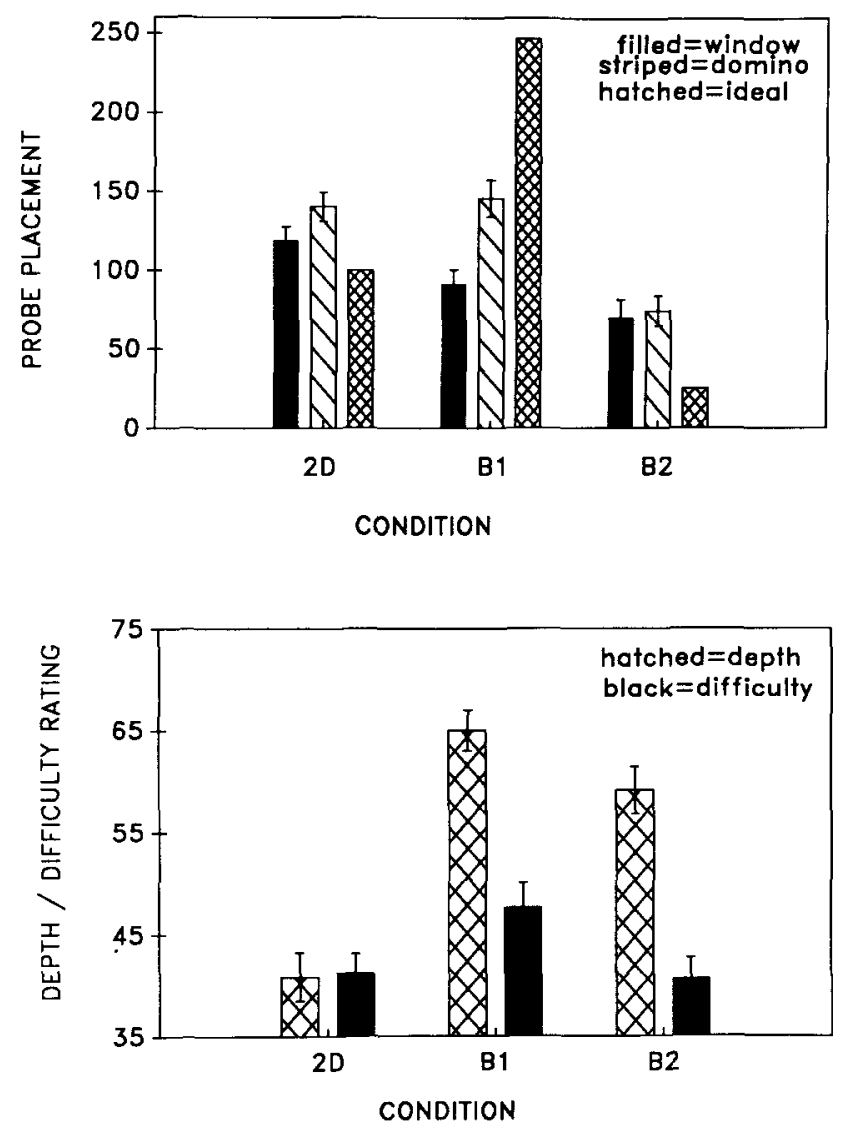

Figure 7. Results of Experiment 2. (Top panel: ideal and actual mean scores for probe placements per condition. In Condition B1 the system is rotated around the external $x$-axis; in B2, around the external $y$-axis. A probe placement of 100 corresponded to circular motion on the projection surface. Two different probe types were used: a window and an additional domino. Bottom panel: Mean depth and difficulty ratings per condition on scales from 0 [in plane/ easy] to 100 [in depth/difficult].) 
placement scores. The results partially met the predictions of the circular path model in three dimension: Stimuli in Condition B2 produced less curvature than predicted by their twodimensional orientation change, $t(11)=2.9, p<.03$. However, for Condition B1, scores did not exceed two-dimensional circularity, and Condition 2D led to curves that curved more than circularity, $t(11)=2.51, p<.03$. In both three-dimensional conditions, curvature differed significantly from that predicted by the circular three-dimensional model; for Condition B1 curvature was $48.1 \%$ of what it should have been, $t(11)=13.86, p<.0001$; for Condition B2 it was $188.6 \%$, $t(11)=4.9, p<.0005$.

Repeated measures ANOVAs were conducted with probe placement, depth rating, and difficulty as dependent variables and condition, gender, and probe style as independent variables. Condition was significant for probe placement, $F(2,20)$ $=34.74, p<.0001$, depth rating, $F(2,20)=8.68, p<.002$, and for difficulty, $F(2,20)=36.48, p<.0001$. Probe style was significant for probe placement only, $F(1,10)=5.59, p$ $<.04$; domino probes produced more curvature (see the top panel of Figure 7). There were no significant effects of gender.

As can be seen in the bottom panel of Figure 7, Conditions $\mathrm{B} 1$ and $\mathrm{B} 2$ received far higher depth ratings than Condition $2 \mathrm{D}, F(1,11)=8.19, p<.02$. That is, observers reported substantial phenomenal depth whenever the apparent-motion display allows for three-dimensional interpretation. Also Condition B1 was judged to be more difficult than Conditions $2 \mathrm{D}$ and $\mathrm{B} 2, F(1,11)=21.11, p<.01$.

\section{Discussion}

The results support the hypothesis of curved trajectories in three dimensions better than that of straight-line trajectories or curves in two dimensions. In all conditions, the perceived paths differed from the straight-line solution. For virtual threedimensional space, the circular path model predicts curvatures that exceed two-dimensional circularity for Condition $\mathrm{B} 1$ and that are below circularity for Condition B2. This prediction was partially met by the mean probe placements for these conditions. Also stimuli belonging to Conditions B1 and $\mathrm{B} 2$ received much higher depth ratings than Condition $2 \mathrm{D}$, which supports the hypothesis that apparent motions occur in three-dimensional space when the stimuli can be interpreted to move at an angle to the projection surface.

However, there is a lack of fit between the placement data and the circular path model applied to three-dimensional space. The apparent-motion trajectories should have been shallower for Condition B2; moreover, they should have curved more for Condition B1 than for Condition 2D, but they did not. The following factors might have prevented observers from perceiving larger curvatures in Condition B1. First, B1 dominos had to travel a much longer distance in three-dimensional space than all other stimuli. Second, a ceiling could have been reached as to how much curvature can be perceived in apparent-motion displays. As discussed early in this article, orientation differences have not been shown to induce path perceptions that greatly exceed circularity. Also the absolute amount of motion required for the Bl stimuli was always larger than for any of the other displays, which may have made judgments very difficult (see the bottom panel of Figure 7). Finally, because perspective was not included in the stimuli, the depth cues provided by orientation change might have been counteracted by the cues of missing perspective.

In essence, Experiment 2 shows that apparent motion can occur in depth. It supports the circular path model better than the straight-line path model, but fails to produce larger curvatures for Condition B1 than for Condition 2D. ${ }^{4}$

\section{Experiment 3}

This experiment was designed to obtain an independent assessment of the orientation of the depth plane in which the curved motion paths were perceived. With this measure, it should become possible to distinguish whether probe placements reflected a perceived path in two-dimensional or in three-dimensional space. We also attempted to enhance the viewing conditions to facilitate the perception of phenomenal depth.

Accordingly, a number of modifications were made: The size of the whole display was reduced by one half. The viewing conditions were optimized by the use of a viewing box. The assessment of perceived depth was improved by introducing an adjustable depth plane. In all other respects, the conditions remained identical to those of Experiment 2.

\section{Method}

Subjects. Twelve observers ( 6 men and 6 women) were tested individually. They were undergraduate students in introductory psychology classes at the University of Virginia and received partial course credit for their participation in the experiment. All subjects had normal or corrected-to-normal vision.

Apparatus. A viewing box was used to facilitate the perception of phenomenal depth by reducing conflicting cues that indicate that the images were two-dimensional projections. The box fit over the terminal and was painted black to reduce the reflection of light. A window inside the box at about two thirds of the total distance from the screen occluded the borders of the computer screen and the inside walls of the viewing box. Thus, the depth cues in the space between the viewpoint and the screen were minimized. At the observer's end, a scuba mask was mounted on the box to prevent light leakage. The viewing distance was $64 \mathrm{~cm}$. A slide with one viewing hole was inserted into the mask to ensure monocular viewing. The apparatus remained unchanged in all other aspects.

Stimuli. Because of the constraints dictated by the fact that clearly distinguishable conditions for $\mathrm{B} 1, \mathrm{~B} 2$, and $2 \mathrm{D}$ had to be created, it was not possible to decrease the necessary curvature or the threedimensional distance for the $\mathrm{B} 1$ dominos relative to the other stimuli.

\footnotetext{
${ }^{4}$ When considering alternative explanations for the results, which support the three-dimensional circular path model, one might be concerned about the following fact: Only for Condition B2 do the two dominos in each display foreshorten as a function of the system's rotation around the $y$-axis. However, Experiment 1 has shown that a foreshortening of the probe (introduced by changing $\alpha_{2}$ ) does not lead to perceived motion paths that are less than circular in two-dimensional norm scores (cf. Figure 5).
} 
However, the amount of absolute motion on the screen could easily be reduced by scaling down the whole display by one half (distance between domino centers was $6.75 \mathrm{~cm}$ ).

The same domino-like objects were used as in Experiment 2, with the exception that they were smaller $(2.6 \times 1.3 \times 0.65 \mathrm{~cm})$ and their centers were closer. In all other respects (blinking sequences, SOA, etc.), the displays and procedures were identical to those in Experiment 2.

After the subject positioned the probe, a key was pressed to turn on the depth plane while the apparent-motion display of blinking dominos remained on the screen. As shown in Figure 8, the plane consisted of a regularly spaced grid. It could be turned, in steps of $5^{\circ}$, around a vertical or a horizontal axis, which went through its center but not around both axes simultaneously. The plane underwent transformations in shape as would the perspective projection of a plane in three dimensions. Observers were asked to make two judgments. First, they had to decide around what axis the depth plane was to be rotated, and then they had to judge the amount of rotation.

Design. The same five sets of stimuli were used as in Experiment 2. The angles of rotation of the system around the external and internal coordinate axes remained unchanged. The two rating scales of difficulty and depth were replaced by the adjustable depth plane. One half of the observers used the window probe; and the other half used the domino probe. Each observer only saw 15 stimuli, that is, one block of trials.

Procedure. The instructions were similar to those given in Experiment 2. Four practice trials were administered to familiarize subjects with the task. A model of three dominos on a plane was shown to demonstrate the task of orienting the depth plane. Subjects were asked to align it with the motion of the domino. After observers had positioned the probe, they had to turn on the depth plane. Observers were encouraged to explore different orientations of the plane until they found that its position coincided with the perceived domino trajectory.

\section{2-D Solution}

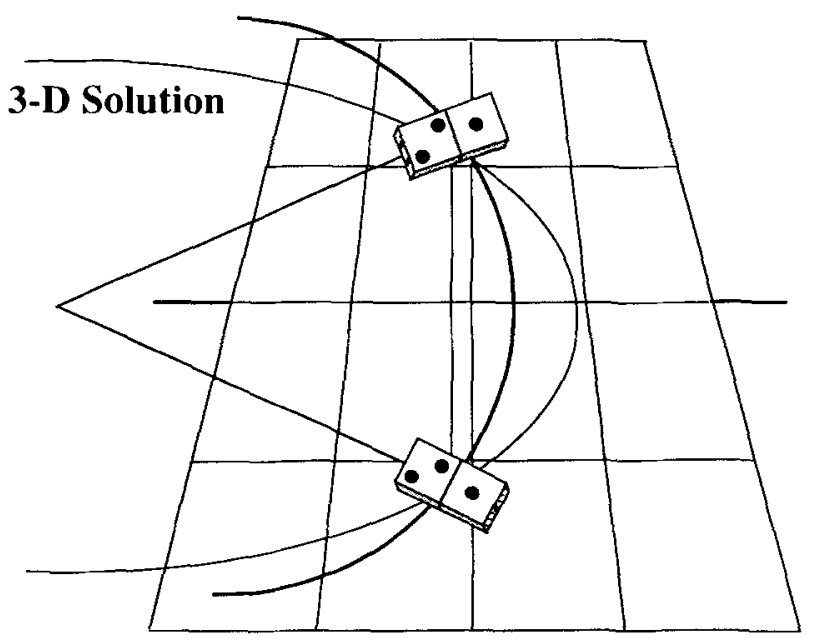

Figure 8. The adjustable depth plane used in Experiment 3 exemplified by a plane orientation corresponding to a B I stimulus (small rotation of the system around the external $x$-axis). (The plane could be rotated interactively around the external $x$ - or $y$-axis in steps of $5^{\circ}$, but not around both axes simultaneously.)

\section{Results}

The mean probe placements resembled the results obtained in Experiment 2 (two-dimensional norm scores were 118.6, 106.4 , and 57.2 for Conditions 2D, B1, and B2, respectively). For Conditions 2D and B1, probe placements did not differ significantly from two-dimensional circularity, but for Condition B2 they did, $t(11)=4.39, p<.001$, although they were still steeper than predicted by the circular three-dimensional model, $t(11)=3.26, p<.008$. A repeated measures ANOVA revealed that the domino probe produced larger curvatures than the window probe, $F(1,10)=10.11, p<.01$; gender had no effect, but condition did, $F(2,20)=10.21, p<.001$.

Figure 9 shows the mean probe placements for those cases in which the accurate depth plane was selected. Conditions $2 \mathrm{D}$ and $\mathrm{B} 1$ did not differ significantly from two-dimensional circularity, but Condition B2 received shallower scores, $t(11)$ $=4.39, p<.001$.

As far as the orientation of the depth plane was concerned, subjects were very accurate in Conditions B1 and B2. In 92\% and $97 \%$ of the cases, respectively, they picked the correct axis; that is, they rotated the depth plane around the horizontal (or vertical) axis when the stimulus pair was actually rotated around the external $x$ - or $y$-axis. However, for Condition $2 \mathrm{D}$, observers performed only at chance level $(33 \%$ correct choice of axis). Figure 10 depicts the judged amount of slant for those trials in which the correct axes had been picked. For individual subjects, the median correlation between actual slant and judged slant was $R=.78$ (ranging from .33 to .90$)$.

A closer look at the probe placements for cases where wrong depth planes were selected for Condition 2D reveals the following: When people thought what they saw was a rotation around the $x$-axis of the system $\left(\beta_{1}>0\right)$, they positioned the probe at a location corresponding to a score of 141.1. However, when they thought the system was rotated around its $y$ axis $\left(\beta_{2}>0\right)$, the score was 98.9. If the motion appeared to be in the picture plane, the average probe placement was 105.4. Thus, apparent-motion trajectories for objects that were correctly identified as moving in two-dimensional space were circular. Trajectories for objects that were misidentified as moving in a plane that was rotated around the system's $x$ axis produced curves that exceeded two-dimensional circularity. These results agree with the predictions of the threedimensional circular path model, and they can explain why the average curvature for Condition $2 \mathrm{D}$ is larger than for a circular path.

\section{Discussion}

The results of Experiment 3 support the hypothesis that observers experience apparent-motion trajectories in threedimensional space. With regard to the depth-plane manipulation, not only were observers able to identify the depth plane in which the stimuli were moving, but they were also quite accurate in judging the relative amount of the plane's slant. Only for Condition 2D did observers have difficulty judging the depth plane. The internal rotation $\left(\alpha_{1}, \alpha_{2}\right)$ of the domino, 


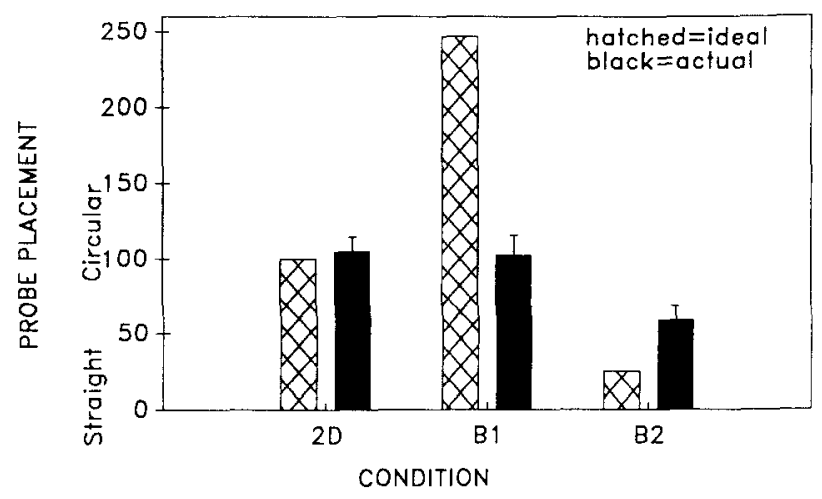

Figure 9. Ideal and actual mean scores for probe placements per condition for cases where correct depth planes had been chosen (Experiment 3).

which was present only for the $2 \mathrm{D}$ stimuli, may have been responsible for this difficulty.

Subjects' probe placements provided some additional support for the circular path model of apparent extended body motions. The fact that the amount of curvature perceived in Condition B2 was less than in Condition B1 is incompatible with the straight-line model and seems to better support the circular path model. For correctly identified 2D stimuli, perceived paths were circular, but the perceived amplitude of curvature for Condition B1 reveals the limits of the circular path model: The observed paths curved much less than the appropriate projection of a three-dimensional circle. The large amount of three-dimensional displacement required for B1 stimuli might be responsible for the lack of curvature even though the reduction of the display size should have produced an effect in the right direction.

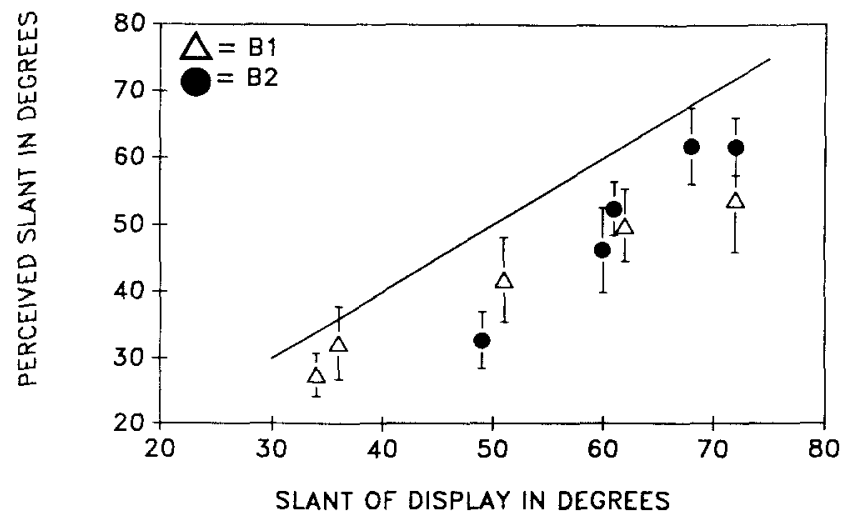

Figure 10. Perceived slant of the apparent-motion trajectory as a function of actual slant of the display in virtual three-dimensional space (Experiment 3). (Triangles denote mean slant ratings for stimuli rotated around the external $x$-axis [Condition $\mathrm{Bl}$ ]; circles denote mean slant ratings for stimuli rotated around the external $y$-axis [Condition B2]. The solid line indicates the actual mean tilt of the display.)

\section{Experiment 4}

The high accuracy at judging depth planes that observers achieved in Experiment 3 could have been based on static cues: Except for Condition 2D, the domino was always oriented parallel to the plane on which it moved (in virtual threedimensional space). This experiment dissociated the domino's orientation from that of its motion plane. If orientation change is sufficient to induce motion paths in three dimensions, observers should still be able to determine the correct depth plane of the apparent motion. However, decreased performance was expected because the task was made more difficult.

\section{Method}

Subjects. Eight observers ( 4 men and 4 women) were tested individually. They were undergraduate students in introductory psychology classes at the University of Virginia and received partial course credit for their participation in the experiment.

Apparatus. Viewing conditions were exactly the same as in Experiment 3 . The display size corresponded to that used in the first two experiments.

Design. Twelve unique stimuli were created: The motion plane in virtual three-dimensional space could be rotated around the system $x$-axis (Condition B1) or $y$-axis (Condition B2) by either $30^{\circ}$ or $60^{\circ}$. Orientation change in three dimensions $(\theta)$ and distance between the domino centers in three dimensions were chosen such that the dominos were always separated by the same distance in two dimensions $(13.5 \mathrm{~cm})$. For each stimulus pair, the orientation of the domino was either parallel to the object ray, rotated around the internal $x^{\prime}$-axis $\left(\alpha_{1}=30^{\circ}\right), y^{\prime}$-axis $\left(\alpha_{2}=30^{\circ}\right)$, or both $\left(\alpha_{1}, \alpha_{2}=30^{\circ}\right)$. Thus, combining all three factors led to 12 unique stimuli ( 2 rotation axes of the system $\times 2$ degrees of system rotation $\times 3$ internal domino orientations). All other parameters (SOA, stimulus shape, etc.) remained the same as in Experiment 3. Only one probe (the third domino) was used to measure apparent curvature because there was no systematic difference between window and domino probes, except that the former produced slightly shallower trajectories.

Procedure. The same instructions were given as in Experiment 3. Subjects were asked to keep in mind that the adjustable depth plane had to be made parallel to the domino's motion (trajectory of its center), not to its orientation.

\section{Results}

Subjects performed well above chance at determining the plane of apparent motion; they picked the correct plane in $59.4 \%$ of the cases for Condition B2 and in $79.7 \%$ for Condition B1. When the correct plane was picked, the mean perceived plane tilt corresponded closely to the actual rotation in depth (for rotations of $30^{\circ}$ and $60^{\circ}$, the plane was tilted on average by $29.4^{\circ}$ and $45.7^{\circ}$, respectively). Dissociating the domino orientation from its motion led to overall shallower trajectories (mean curvature in two-dimensional norm scores was 81.2 and 53.7 for Conditions B1 and B2). Condition B1 differed significantly from its circular three-dimensional solution, $t(7)=10.94, p<.0001$, but Condition $\mathrm{B} 2$ did not. An interaction was found between condition and internal domino orientation: If the system was rotated around the $y$-axis (Con- 
dition B2) and the domino was rotated internally around $y^{\prime}$ or around $x^{\prime}$ and $y^{\prime}$, more shallower curves were reported than in all other cases, $F(3,18)=3.75, p<.03$.

\section{Discussion}

Dissociating the orientation of the stimulus from the depth plane of its virtual motion in three dimensions reduced the amount of perceived curvature and the accuracy rate of judging the depth plane of apparent motion. However, subtle orientation differences are sufficient to induce apparent trajectories in depth. Observers are fairly reliable at determining axis and degree of rotation of the suggested depth plane.

\section{Experiment 5}

Experiments 1 through 4 yielded apparent motion in depth that followed curved trajectories. However, models that predict circular three-dimensional paths could not be strictly corroborated, because empirical curvature was less than predicted in those cases where it should have exceeded twodimensional circularity (Condition B1). By introducing a somewhat exaggerated perspective into the displays, we attempted to push perceived curvature toward three-dimensional circularity.

\section{Method}

Subjects. Eight observers ( 4 men and 4 women) were tested individually. They were undergraduate students in introductory psychology classes at the University of Virginia and received partial course credit for their participation in the experiment.

Apparatus. The same apparatus was used as in Experiment 4, except that the viewing box (reduction screen) was removed. Viewing distance was reduced to $25 \mathrm{~cm}$ to be compatible with the polar perspective introduced to the stimuli. The display size corresponded to that used in the first two experiments. Stimuli were viewed monocularly in a dark room.

Design. Nine unique stimuli were created. The motion plane in virtual three-dimensional space could be rotated around the system $x$-axis (Condition B1) or the $y$-axis (Condition B2) by either $30^{\circ}, 45^{\circ}$, or $60^{\circ}$, or it could stay parallel to the picture plane (Condition $2 \mathrm{D}$ ). To keep the displacement of object centers on the screen constant $(13.5 \mathrm{~cm})$ and the projected orientation difference between the objects at $30^{\circ}$, three-dimensional $\Theta$ s were $33.7^{\circ}, 39.2^{\circ}$, and $49.1^{\circ}$ for Condition $B 1$ and $26.6^{\circ}, 22.2^{\circ}$, and $16.1^{\circ}$ for Condition B2. For stimuli in Condition 2D, $\theta$ s were $16.1^{\circ}, 32.7^{\circ}$, and $49.1^{\circ}$. The stimuli were rotated slightly around their internal $x^{\prime}$ - and $y^{\prime}$-axes to make three surfaces visible $\left(\alpha_{2}\right.$ and $\alpha_{2}$ were $\left.10^{\circ}\right)$. This led to nine stimuli ( 3 conditions $\times 3$ degrees of tilt/ $\theta$ s). All other parameters (SOA, stimulus shape, etc.) remained the same as in Experiment 4. All stimuli were drawn as polar projections corresponding to a viewing distance of $25 \mathrm{~cm}$. This produced clearly noticeable differences in size and shape of the stimuli.

Procedure. The same instructions were given as in Experiment 4.

\section{Results}

Introducing perspective representation did not lead to more pronounced apparent curvature. Mean perceived curvature in two-dimensional norm scores were 83.3 for Condition 2D,
113.5 for Condition B1, and 54.8 for Condition B2. Even though Condition B1 produced steeper curves than Condition $2 \mathrm{D}, F(1,6)=64.8, p<.0002$, the curves did not differ significantly from a circular path in two dimensions, $t(7)=$ $1.19, p>.27$. As expected, curvature for Condition $\mathrm{B} 2$ was less than two-dimensional circularity, $t(7)=10.93, p<.0001$. Depth-plane adjustments were accurate in $100 \%$ of the cases for Condition B1, $85.4 \%$ for Condition B2, and $45.8 \%$ for Condition 2D.

\section{Discussion}

Introducing noticeable perspective projection into the stimulus displays was not sufficient to induce apparent motions that would correspond to circular trajectories in three-dimensional space. Even though observers clearly indicated that they saw curved motions in depth, perceived curvature did not exceed two-dimensional circularity. There appears to be a limit as to the amount of curvature that orientation change can cause in apparent motion.

\section{Conclusion}

The perception of curved trajectories for apparent extended body motions in the picture plane generalizes to similar motion paths in depth. Stimulus orientation alone can induce motion paths in three-dimensional space. These paths are always curved, but only in some cases do they follow circular three-dimensional motions. These findings correspond to results obtained for extended apparent body motions in the picture plane; that is, paths appear curved but often they are less than circular (McBeath, in press; Proffitt et al., 1988).

The experiments provided further evidence that apparent extended body motions are inadequately described by models that propose linear centroid trajectories with concomitant rotations, motions that result from dynamic minimization (Carlton \& Shepard, 1990a, 1990b) as well as from kinematic minimization of common and relative object motions (Cutting \& Proffitt, 1982). Alternatively, the results seem to suggest that, over a wide range of parameters, apparent-motion paths in depth cannot support a rigorous circular path model.

For the two-dimensional conditions, where the dominoshaped test stimuli were oriented parallel to the screen, perceived trajectories were close to circular. For the conditions that allowed for depth interpretations, observers not only reported the apparent motions to occur in the appropriately oriented depth planes, but they were also quite accurate at judging the amount of slant. For stimuli that should produce elliptical projections of little curvature, subjects responded accordingly. For stimuli that should produce steeper curves, the results indicate that the perceived trajectories fall far short of three-dimensional circularity. Under optimal conditions, perceived trajectories can come very close to projections of three-dimensional circles, whereas for other parameter settings the perceived paths deviate from circularity toward the straight-line solution but always remain curved.

Future research could modify the stimulus displays so as to optimize the perception of motion paths in depth. For ex- 
ample, stereopsis could be introduced in addition to orientation change and perspective because its absence might have produced information in conflict with the present cues for depth and thus diminished the perception of three-dimensional circular paths. However, doubts remain as to whether the circular path model can do more than approximate the apparent-motion trajectories of extended bodies by predicting the upper bound of curvature that will be perceived.

The results allow some conclusions with respect to the nature of internalized constraints that govern the perception of moving extended objects. For apparent motion in depth, the internalized principles do not correspond to dynamic physical constraints. The representations of apparent extended body motions appear to reflect processes that are more sensitive to kinematics (Shepard, 1984) or information minimization (Restle, 1979) than they are to natural dynamics.

\section{References}

Attneave, F., \& Block, G. (1973). Apparent movement in tridimensional space. Perception \& Psychophysics, 13, 301-307.

Bundesen, C., Larsen, A., \& Farrell, J. E. (1983). Visual apparent movement: Transformations of size and orientation. Perception, $12,549-558$.

Carlton, E. H., \& Shepard, R. N. (1990a). Psychologically simple motions as geodesic paths: I. Asymmetric objects. Journal of Mathematical Psychology, 34, 127-188.

Carlton, E. H., \& Shepard, R. N. (1990b). Psychologically simple motions as geodesic paths: II. Symmetric objects. Journal of Mathematical Psychology, 34, 188-228.

Cutting, J. E., \& Proffitt, D. R. (1982). The minimum principle and the perception of absolute, common, and relative motions. Cognitive Psychology, 14, 211-246.

Farrell, J. E. (1983). Visual transformations underlying apparent movement. Perception \& Psychophysics, 33, 85-92.

Farrell, J. E., \& Shepard, R. N. (1981). Shape, orientation, and apparent rotational motion. Journal of Experimental Psychology: Human Perception and Performance, 7, 477-486.

Foster, D. H. (1975). Visual apparent motion and some preferred paths in the rotation group $\mathrm{SO}(3)$. Biological Cybernetics, 18, 8189.

Foster, D. H. (1978). Visual apparent motion and the calculus of variations. In E. L. J. Leeuwenberg \& H. F. J. M. Buffart (Eds.), Formal theories of visual perception (pp. 67-82). New York: Wiley. Gerbino, W. (1984). Low-level and high-level processes in the perceptual organization of three-dimensional apparent motion. Perception, 13, 417-428.

Green, M., \& Odom, V. (1986). Correspondence matching in apparent motion: Evidence for three-dimensional spatial representation. Science, 233, 1427-1429.

Kolers, P. A., \& Pomerantz, J. R. (1971). Figural change in apparent motion. Journal of Experimental Psychology, 87, 99-108.

McBeath, M. K. (in press). Comparison of four models that describe path of apparent motion. Journal of Experimental Psychology: Human Perception and Performance.

McBeath, M. K., \& Shepard, R. N. (1989). Apparent motion between shapes differing in location and orientation: A window technique for estimating path curvature. Perception \& Psychophysics, 46, 333-337.

Mori, T. (1982). Apparent motion path composed of a serial concatenation of translations and rotations. Biological Cybernetics, 44, 31-34.

Proffitt, D. R., Gilden, D. L., Kaiser, M. K., \& Whelan, S. M. (1988). The effect of configural orientation on perceived trajectory in apparent motion. Perception \& Psychophysics, 43, 465-474.

Restle, F. (1979). Coding theory of the perception of motion configurations. Psychological Review, 86, 1-24.

Shepard, R. N. (1984). Ecological constraints on internal representation: Resonant kinematics of perceiving, imagining, thinking, and dreaming. Psychological Review, 91, 417-447.

Shepard, R. N., \& Judd, S. A. (1975). Perceptual illusion of rotation of three-dimensional objects. Science 191, 952-954.

Shepard, R. N., \& Metzler, J. (1971). Mental rotation of threedimensional objects. Science, 171, 701-703.

Shepard, R. N., \& Zare, S. L. (1983). Path-guided apparent motion. Science, 220, 632-634.

Wertheimer, M. (1912). Experimentelle Studien über das Sehen von Bewegung [Experimental studies about motion perception.] Zeitschrift für Psychologie, 61, 161-265.

White, D.G., Wenderoth, P., \& Curthoys, I. S. (1979). The importance of pattern information for the resolution of depth-ambiguous apparent motion. Perception \& Psychophysics, 26, 355-362.

\section{Appendix}

\section{Method Used to Create the Stimuli}

Because rotating a two-dimensional stimulus pair into depth modifies the projected orientation and distance between the two objects (displacement), two modifications had to be made to create stimuli that produced a given displacement and orientation of its two-dimensional projection. First, three-dimensional orientation had to be altered; second, three-dimensional size had to be changed. These two modifications will be explained in detail later, first for stimuli rotated around the external $x$-axis (Condition B1) and then for stimuli rotated around the external $y$-axis (Condition B2).

\section{Rotation Around External $x$-Axis $\left(\beta_{1}>0\right)$ for Stimuli in Condition B1}

A given stimulus pair $i$ in the picture plane (Condition 2D) transforms as follows if it is rotated around the $x$-axis:

$$
\cos \left(\beta_{1 j}\right) \times \tan \left(\Theta_{j}\right)=\cos \left(\beta_{1 i}\right) \times \tan \left(\Theta_{i}\right),
$$

where $\beta_{1 i}$ and $\beta_{1 j}$ are the angles of rotation around the external $x$-axis for the two stimulus pairs $i$ (in two dimensions) and $j$ (in three dimensions). $\theta$ is the angle between the object rays and the system axis (cf. Figure 4). 
For example, a two-dimensional stimulus pair $i$ with $\theta_{i}=30$ and $\beta_{1 i}=0$ would have to be modified as follows to keep two-dimensional orientation difference and displacement constant. If $\beta_{1 j}$ (the amount of rotation around the system axis) were selected to be $60^{\circ}$, then

$$
\tan \left(\theta_{j}\right)=\frac{\cos (0) \times \tan (30)}{\cos (60)}=1.15 \text {. }
$$

It follows that $\theta_{j}=49.1$.

To keep the two-dimensional displacement constant, the threedimensional displacement has to be transformed according to

$$
D_{j}=\frac{D_{i}}{\cos \left(\beta_{1 j}\right)}
$$

where $D_{j}$ is the linear distance in three-dimensional space between the two dominos to project a displacement of $D_{i}=13.5 \mathrm{~cm}$ in twodimensional space. For stimuli in Condition B1, the three-dimensional displacement had to be made larger for all displays. For instance, for this example:

$$
D_{j}=\frac{13.5 \mathrm{~cm}}{\cos (60)}=27.0 \mathrm{~cm} .
$$

\section{Rotation Around External $y$-Axis $\left(\beta_{2}>0\right)$ for Stimuli in Condition B2}

If a given stimulus pair $i$ of Condition 2D was rotated around the external $y$-axis to create a stimulus pair $k$ (Condition B2), orientation between the dominos in three dimensions $\left(\Theta_{k}\right)$ had to be modified to keep projected orientation constant:

$$
\tan \left(\theta_{k}\right)=\cos \left(\beta_{2 k}\right) \times \tan \left(\theta_{i}\right) .
$$

The displacement of the objects did not need to be modified $\left(D_{k}=\right.$ $\left.D_{i}\right)$.

Received June 4, 1990

Revision received December 19, 1990 Accepted December 21, 1990

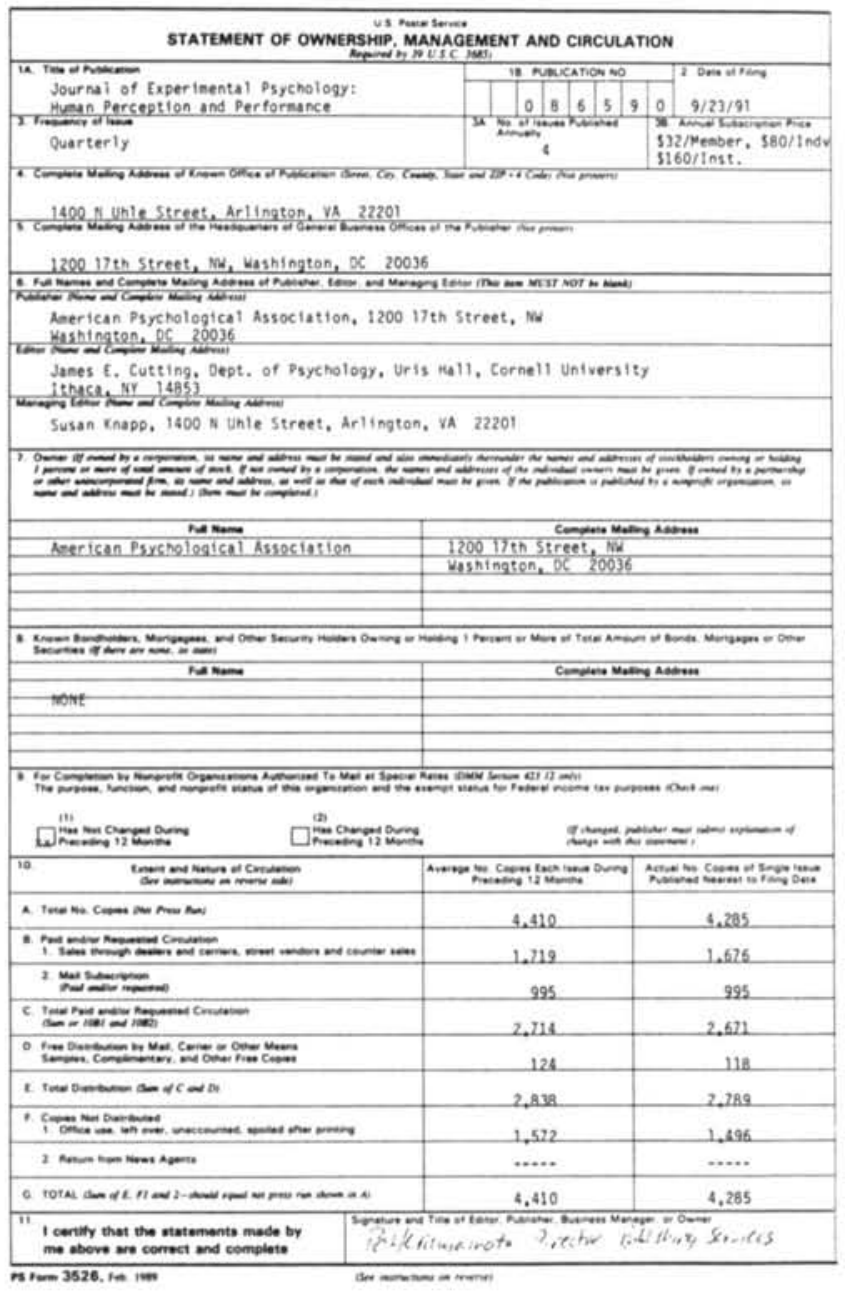

